\title{
Gene therapy of renal cancer using recombinant adeno-associated virus encoding human endostatin
}

\author{
ERLIN SUN ${ }^{1}$, RUIFA HAN ${ }^{1}$ and BINGXIN LU ${ }^{2}$ \\ ${ }^{1}$ Tianjin Key Laboratory of Urology Basic Science, Tianjin Institute of Urology, \\ The Second Hospital of Tianjin Medical University, Tianjin 300211; ${ }^{2}$ Department of Urology, Tianjin Nankai Hospital \\ (Tianjin Hospital of Integrated Traditional Chinese and Western Medicine), Tianjin 300100, P.R. China
}

Received April 26, 2016; Accepted December 20, 2017

DOI: $10.3892 / \mathrm{ol} .2018 .9036$

\begin{abstract}
Renal cell carcinoma (RCC) is characterized by robust angiogenesis during tumor development. Various therapies are not able completely eradicated tumor relapse. The present study targeted angiogenesis and developed a recombinant adeno-associated virus ( $\mathrm{AAAV}$ ) vector containing human endostatin gene for human kidney cancer gene therapy. Prophylactic and therapeutic RCC models were established in nude mice by subcutaneous inoculation of RCC cells and intra-muscular or intra-tumor injection of rAAV-Endostatin. The growth of xenograft tumors was evaluated by tumor volume and weight. The microvessel density (MVD) was used to measure the anti-angiogenesis effect of rAAV-Endostatin. The toxic effect of rAAV-Endostatin was also examined. In the therapeutic model, tumor-bearing mice with rAAV-Endostatin intra-tumor injection demonstrated slow tumor growth $(32.63 \pm 9.75)$ compared with control groups with intratumoral rAAV-enhanced yellow florescent protein (EYFP) injections $(21.50 \pm 11.42)$ and the RPMI-1640 group (21.75 \pm 10.48 days, for tumors to reach $\sim 300 \mathrm{~mm}^{3}$ ). MVD of the xenografts treated with rAAV-Endostatin was $8.30 \pm 3.14 / 0.739 \mathrm{~mm}^{2}$ whereas that of control groups was $13.87 \pm 4.09 / 0.739 \mathrm{~mm}^{2}$ (rAVV-EYFP) and $13.76 \pm 3.50 / 0.739 \mathrm{~mm}^{2}$ (RPMI-1640). No significant side effects associated with rAAV-endostatin use were identified in the vital organs. rAAV-Endostatin demonstrated significant anti-angiogenesis and antitumor activities. It may serve as an effective agent for renal cancer gene therapy.
\end{abstract}

\section{Introduction}

It has been established that tumor growth depends on angiogenesis, a process of continued expansion of endothelial cells from pre-existing blood vessels $(1,2)$. Tumors in situ, which are

Correspondence to: Dr Bingxin Lu, Department of Urology, Tianjin Nankai Hospital (Tianjin Hospital of Integrated Traditional Chinese and Western Medicine), 6 Changjiang Road, Tianjin 300100, P.R. China

E-mail: bingxinlu01@sohu.com

Key words: renal cancer, gene therapy, endostatin, angiogenesis
$<3 \mathrm{~mm}$ in diameter, exist in a pre-vascular state and are limited in their ability to grow without perfusion from the blood supply (1). The recruitment of novel blood vessels increases the availability of oxygen and metabolites to tumors. In addition, this newly formed vasculature facilitates the escape of tumor cells to distant regions of the body (3). The inhibition of tumor angiogenesis, therefore, is an important potential therapy for solid tumors. Therapies designed to inhibit novel blood vessel formation have advantages, in that they target the genetically stable endothelial cells with resistance to anti-angiogenesis therapy (4).

Multiple endogenous angiogenic inhibitors, including thrombospondin, interferon $\alpha$, platelet factor 4 , PEX-the C-terminal fragment of matrix metalloproteinase 2-angiostatin and endostatin, have been characterized and demonstrated to elicit antitumor effects $(5,6)$. Among these, endostatin, a $20 \mathrm{kDa}$ C-terminal proteolytic fragment of collagen XVIII, has received the greatest attention. Not only was identified to be a potent inhibitor of angiogenesis in vitro, but also has been suggested to have significant antitumor effects in a variety of preclinical tumor models (7). Externally administered endostatin was progressed quickly into clinical trials; however, clinical development was halted due to limited efficacy and problems with protein formulation and application (8). Due to this, gene therapy applying endostatin is attractive. Anti-angiogenic gene therapies involving non-viral methods, including plasmid or naked DNA, and viral strategies, including adenoviruses, adeno-associated viruses, retro-oncoviruses and lentiviruses, have been employed in numerous rodent tumor models (9-11). Recombinant adeno-associated vector (rAAV) is a replication-deficient, non-pathogenic vector belonging to the group of human parvoviruses (12). These vectors have great potential for cancer gene therapy, as they transduce dividing and non-dividing cells, are less immunogenic compared with other vectors, and are able to integrate into the host genome, allowing long-term transgene expression (13). However, only a small number of previous studies have used rAAVs to deliver anti-angiogenic genes for cancer therapy: Davidoff et al (14) demonstrated that the portal vein injection of an rAAV encoding soluble fetal liver kinase 1 (Flk-1) resulted in FLK-1 protein expression from liver for up to 6 months, and that this vector was effective in reducing tumor vessel density and the subsequent size of SK-NEP-1 tumors in subcutaneous 
and orthotopic mouse models. It has also been demonstrated that a single injection of an rAAV encoding endostatin provides long-term expression of endostatin (15), and that the rAAV may enhance the treatment efficacy of radiation in a human colorectal tumor model (HT29) (16). Other studies demonstrated that the systemic use of rAAVs encoding endostatin may inhibit angiogenesis, growth and metastases in pancreatic and ovarian cancers (17-19). In the present study, an rAAV encoding human endostatin was developed, and its antitumor activity in a xenograft renal tumor model was evaluated.

\section{Materials and methods}

Plasmids and viral construction. Multiple plasmids were used, including pIRES-Endo containing human IgG-Endostatin sequence and the AAV helper-free system (Clontech Laboratories, Inc., Mountainview, CA, USA), for example: pAAV-multiple cloning site (MCS), pCMV-MCS, pRC and pHelper. The pHelper, which contains adenovirus-derived genes, was used for viral packaging to avoid adenovirus contamination. Plasmids were prepared by standard alkaline lysis $(0.2 \mathrm{~N} \mathrm{NaOH} / 1.0 \%$ sodium dodecyl sulfate) procedure followed by ethanol precipitation (2.5 volumes) using the Plasmid Isolation (Alkaline Lysis) kit (cat. no. BE-310; G-Biosciences, St. Louis, MO, USA) according to the manufacturer's protocol. The IgG-Endostatin was amplified using polymerase chain reaction from the pIRES-Endo plasmid containing human IgG-Endostatin (a gift from Professor Xiao-Yan Wen, University of Toronto, Toronto, Canada) using a Taq PCR kit (cat. no. E5000S; New England BioLabs, Inc., Ipswich, MA, USA). The sequence of the forward primer was GACATCGATATGAAATGCAGCTGGGTTATC (ClaI site underlined) and the reverse primer was TATGGATCCCTA CTTGGAGGCAGTCATG (BamHI site underlined). The PCR conditions were designed as follows: Initial denaturation at $95^{\circ} \mathrm{C}$ for $30 \mathrm{sec}, 30$ cycles of $95^{\circ} \mathrm{C}$ for $30 \mathrm{sec}, 55^{\circ} \mathrm{C}$ for $30 \mathrm{sec}$ and $72^{\circ} \mathrm{C}$ for $1 \mathrm{~min}$ followed by final extension at $72^{\circ} \mathrm{C}$ for $3 \mathrm{~min}$. Subsequent to sequencing, the target segment was cloned into pCMV-MCS to construct the pCMV-Endo plasmid. To avoid inverted terminal repeats rearrangement, pCMV-Endo was digested by NotI and the expression cassette containing IgG-Endostatin was sub-cloned into pAAV-MCS to construct the pAAV-Endo vector plasmid.

Preparation of rAAV-endostatin. The 293 cells (American Type Culture Collection Manassas, VA, USA) were grown in Dulbecco's modified Eagle's medium (DMEM; Thermo Fisher Scientific, Inc., Waltham, MA, USA) supplemented with $5 \%$ heat-inactivated fetal bovine serum (FBS; Japan Bioserum Co., Ltd., Hiroshima, Japan) to $\sim 80 \%$ confluence, then digested using $0.25 \%$ trypsin (Thermo Fisher Scientific, Inc.) and counted using a hemocytometer and a light microscope (magnification, $\mathrm{x} 20$ ), and then co-transfected with pAAV-Endo, pRC and pHelper to package rAAV-Endostatin $\left(0.3 \mu \mathrm{g} / \mathrm{each}\right.$ in $25 \mu \mathrm{l}$ DMEM medium) using Lipofectamine ${ }^{\mathrm{TM}}$ transfection reagent (Thermo Fisher Scientific, Inc.) according to the manufacturer's protocol. All cell lines used in the present study were cultured in a humidified incubator with $5 \% \mathrm{CO}_{2}$ at $37^{\circ} \mathrm{C}$. The parameter of the gene pulser used for electroporation was $960 \mu \mathrm{F} / 245 \mathrm{~V}$, for a cuvette with a gap of $0.4 \mathrm{~cm}$. A total of $72 \mathrm{~h}$ later, cells were frozen and thawed at $-20^{\circ} \mathrm{C}$ and $37^{\circ} \mathrm{C}$ for 3 cycles, and then $100 \mu \mathrm{l}$ viral solution was added to fresh 293 cells to prepare the second viral generation. After $72 \mathrm{~h}$, the procedure was repeated to prepare the third viral generation. The viral solution was centrifuged at 1,000 $\mathrm{x} g$ in room temperature for $30 \mathrm{~min}$ followed by $8,000 \mathrm{x} \mathrm{g}$ at $4^{\circ} \mathrm{C}$ for $1 \mathrm{~h}$. Following purification using the Adenovirus Purification kit (cat. no. 631533; Clontech Laboratories, Inc.), the virus was verified using transmission electron microscopy as previously described (20) (magnification, x30,000) and assayed using the human adeno-associated virus ELISA kit (cat. no. MBS260177; MyBioSource, San Diego, CA, USA).

The human RCC OS-RC-2 cell line (American Type Culture Collection) was maintained in the Tianjin Institute of Urology, and cultured in DMEM supplemented with $10 \%$ heat-inactivated FBS (Gibco; Thermo Fisher Scientific, Inc., Waltham, MA, USA). Cultured OS-RC-2 cells ( $80 \%$ confluence) were infected with $\mathrm{rAAV}-$ Endostatin $\left(\mathrm{MOI}=10^{5}\right)$ at $4^{\circ} \mathrm{C}$. After $24 \mathrm{~h}, 1.0 \times 10^{6} \mathrm{OS}-\mathrm{RC}-2$ cells were plated in 6 -well dishes for $24 \mathrm{~h}$ at $37^{\circ} \mathrm{C}$. The supernatant was then collected by centrifuging at $300 \mathrm{x} \mathrm{g}$ for $7 \mathrm{~min}$ at room temperature and assayed for endostatin secretion by ELISA as aforementioned. Human umbilical vein endothelial cells (HUVEC) (American Type Culture Collection) chemotactic movement was analyzed by Transwell assay as previously described (21) to evaluate recombinant endostatin activity.

RCC tumor model. $15 \mathrm{BALB} / \mathrm{c}$ nude mice (Shanghai SLAC Laboratory Animal Co. Ltd., Shanghai, China) at age of 5-6 weeks and a weight of $25 \mathrm{~g}$ were housed in $12 / 12 \mathrm{~h}$ light/dark cycle specific pathogen free conditions at $24 \pm 2{ }^{\circ} \mathrm{C}$ and a humidity of $1.5 \% \mathrm{CO}_{2}$ with free access to food and water. All mice were used in the following 4 experiments: i) Prophylactic model, in which 24 nude mice were randomly divided into 3 groups and injected with 3 doses of $1.0 \times 10^{11}$ rAAV-Endostatin viral particles (v.p), 1.0 $10^{11}$ rAAV-EYFP v.p, or RPMI-1640 medium (Thermo Fisher Scientific, Inc.), respectively, into the right hind leg tibialis anterior muscles at 1 week intervals. At 1 week following the final immunization, $1.0 \times 10^{6}$ OS-RC-2 cells were inoculated subcutaneously on the dorsal surface of mice. The xenograft formation rate, size and weight were monitored; ii) therapeutic model, in which $\mathrm{BALB} / \mathrm{c}$ nude mice were inoculated with $1 \times 10^{6}$ OS-RC-2 cells through subcutaneous injection. A total of 10 days subsequent to tumor growth reaching $\sim 5 \mathrm{~mm}, 3$ doses of rAAV-Endostatin or control rAAV were administered via intratumoral injection into the RCC tumor. The xenograft tumor was measured every day until tumor size reached $\sim 300 \mathrm{~mm}^{3}$. Mice were then sacrificed by cervical dislocation and xenografts were assayed for microvessel density (MVD) by immunohistochemical staining. Briefly, xenografts were fixed in $4 \%$ formalin overnight at $4{ }^{\circ} \mathrm{C}$ followed by paraffin embedding. Tissues were then cut into $10 \mu \mathrm{m}$-thick sections in, air dried overnight at room temperature, and fixed in acetone for $10 \mathrm{~min}$ at room temperature. Slides were allowed to air dry for $1 \mathrm{~h}$ and washed three times for $5 \mathrm{~min}$ each in phosphate-buffered saline (PBS; pH 7.4). Samples were then blocked with PBS containing $0.1 \%$ bovine serum albumin (Thermo Fisher Scientific, Inc.) and 3\% human 
A

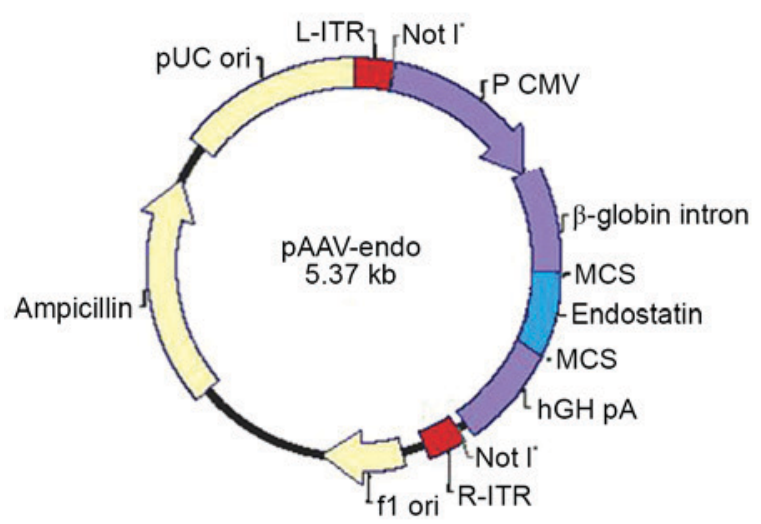

B

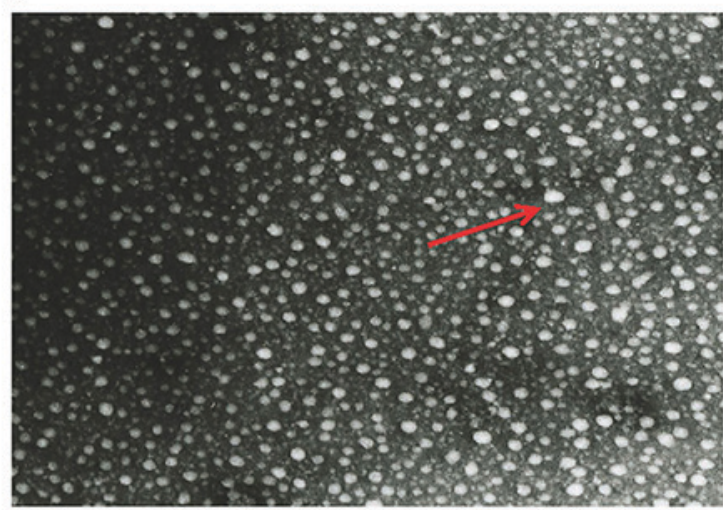

C

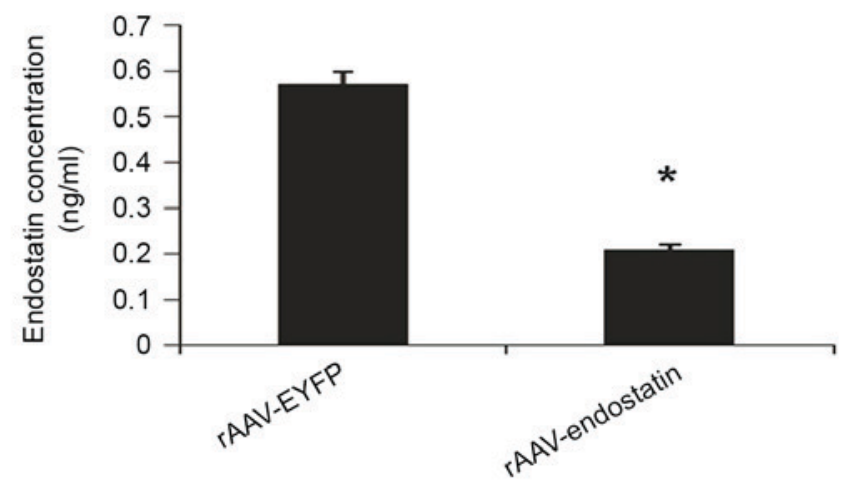

Figure 1. Construction, production and infection of rAAV-Endostatin. (A) Construction of rAAV-endostatin vector. The endostatin gene was inserted into the MCS of pAAV vector; OS-RC-2 cells infected with rAAV-YRFP. (B) The rAAV particles in tumor cells are indicated by transmission electron microscopy (magnification, x80,000). (C) Endostatin production in OS-RC-2 cells infected with rAAV-Endostatin was analyzed by ELISA. Comparison between RCC-rAAV-RYFP cells and OS-RC-2-rAAV-endostatin cells was performed using analysis of variance. "P $<0.05$. MCS, multiple cloning site; ITR, inverted terminal repeats; AAV, adenovirus-associated vector; EYFP, enhanced yellow florescent protein; ori, origin of replication.

serum albumin (Thermo Fisher Scientific, Inc.) for at least $30 \mathrm{~min}$ at room temperature. Subsequently, all slides were incubated with rabbit polyclonal antibody against CD31 (1:50 dilution) (cat. no. ab28364; Abcam, Cambridge, MA, USA) at $4^{\circ} \mathrm{C}$ overnight. Following washing with tris-buffered saline with Tween-20 $(0.1 \%)$ three times, biotinylated goat anti-rabbit IgG (1:1,000; Jackson Immuno Research Laboratories, Inc., West Grove, PA, USA) was added into the sections and incubated for $30 \mathrm{~min}$ at room temperature followed by incubation with Strepavidin-horseradish peroxidase (Sigma-Aldrich; Merck KGaA, Darmstadt, Germany) for $30 \mathrm{~min}$ at room temperature. The staining was visualized using diaminobenzidine (ScyTek Laboratories, Inc., Logan, UT, USA) under a light microscope (x200). iii) 24 nude mice were injected with $1.0 \times 10^{11}$ rAAV-Endostatin v.p. A total of 3 mice were sacrificed every 10 days and the endostatin concentration in serum was assayed by ELISA as aforementioned. The rAAV-EYFP was used as control; and iv) 8 mice were injected with $1.0 \times 10^{11} \mathrm{rAAV}$-Endostatin v.p and sacrificed after 8 weeks for heart and encephalon collection. Histological hematoxylin (10\%) and eosin (1\%) (H\&E) staining as described previously (22) and transmission electron microscopy (magnification $\mathrm{x} 30,000$ ) as aforementioned, were performed to determine whether rAAV-Endostatin caused ischemia or other pathological changes in these organs.
Statistical analysis. The data were analyzed using one-way analysis of variance and Tukey's tests to detect any significance. $\mathrm{P}<0.05$ was considered to indicate a statistically significant difference. All data are presented as mean \pm standard error of the mean. Statistical analyses were performed using SAS statistical software v9.3 (SAS Institute, Cary, NC, USA).

\section{Results}

Construction of $r A A V$-endostatin vector and production of $r A A V$ virus. The pAAV-endostatin vector plasmid was constructed by sub-cloning of the pCMV-endostatin vector, which was established by insertion of endostatin fragment (Fig. 1A) and verified by sequencing. rAAV-Endostatin was packaged and demonstrated the normal viral pattern under electron microscopy. The titer of viral preparation was $1.0 \times 10^{12} \mathrm{v} . \mathrm{p} / \mathrm{ml}$ by ELISA.

Following infection with rAAV-Endostatin or rAAV-YRFP with MOI of $10^{5}, \sim 95 \%$ OS-RC-2 cells were infected (Fig. 1B). To measure endostatin production, OS-RC-2-rAAV-Endostatin or control cells were cultured for $72 \mathrm{~h}$, and supernatant were collected for ELISA assay. OS-RC-2 cells infected with $\mathrm{rAAV}$-Endostatin produced $54.09 \mathrm{ng} / \mathrm{ml}$ recombinant endostatin in the culture supernatant compared with $0.4 \mathrm{ng} / \mathrm{ml}$ RCC-rAAV-RYFP cells (Fig. 1C). 
A
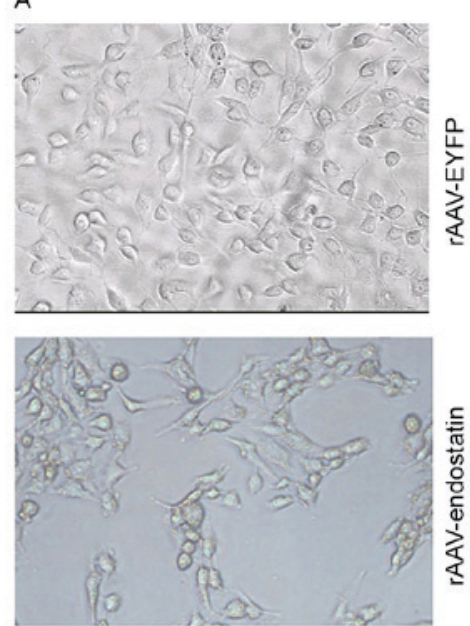

B

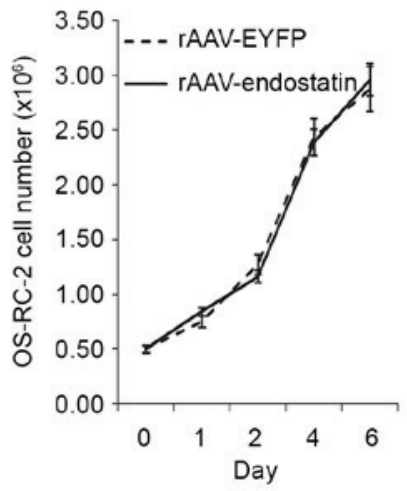

C

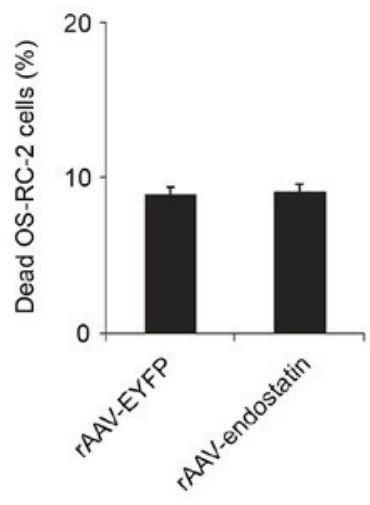

Figure 2. rAAV-endostatin infection did not affect tumor cell proliferation and apoptosis in renal cell carcinoma cells. (A) The morphology of OS-RC-2 cells infected with rAAV-endostatin and rAAV-YRFP was analyzed. The two cell groups exhibited epithelial-like growth, without significant morphological changes (magnification, $\mathrm{x} 400$ ). (B) Cell proliferation and (C) cell death of OS-RC-2-endostatin and OS-RC-2-YRFP cells were compared by analysis of variance, and no difference between the two cell lines was observed. rAAV, recombinant adenovirus-associated vector; EYFP, enhanced yellow florescent protein.

A
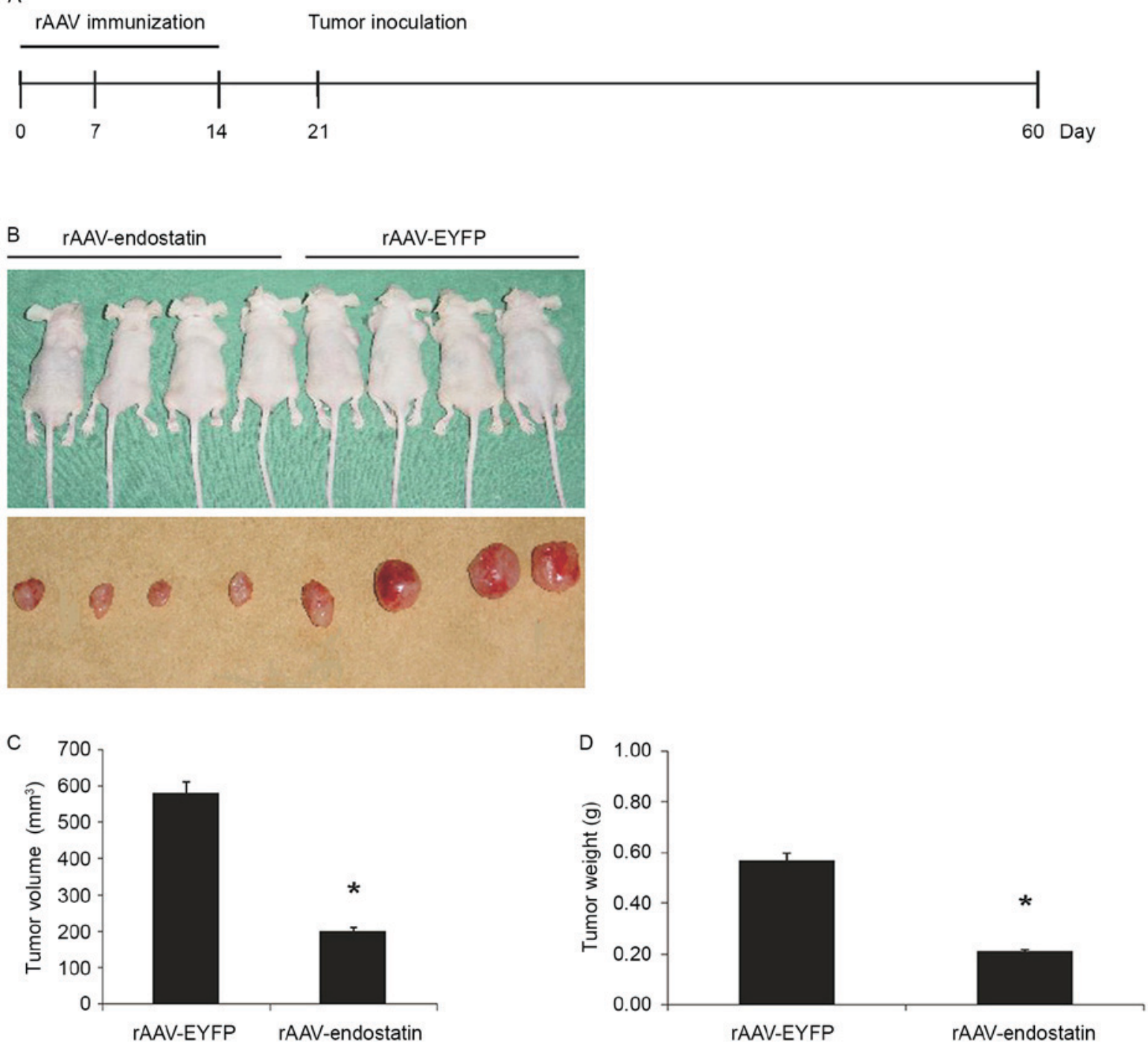

Figure 3. Prophylactic effect of rAAV-endostatin in RCC tumor development. (A) The prophylactic immunization model. rAAV-endostatin inhibited bladder cancer occurrence and development effectively. (B) A total of 4 weeks following subcutaneous inoculation, OS-RC-2 tumors with rAAV-endostatin immunization developed 50\% xenograft tumors but the controls was 100\% (8/8 mice), and the (C) volume and (D) weight of tumors were all less compared with the controls (analyzed by analysis of variance). rAAV, recombinant adenovirus-associated vector; EYFP, enhanced yellow florescent protein. ${ }^{*} \mathrm{P}<0.05$. 
A

Tumor inoculation rAAV intratumor injection

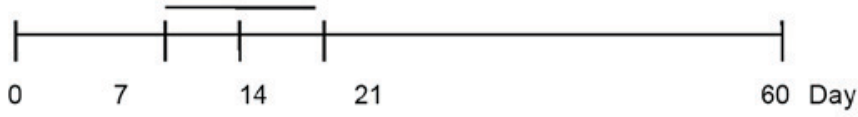

C

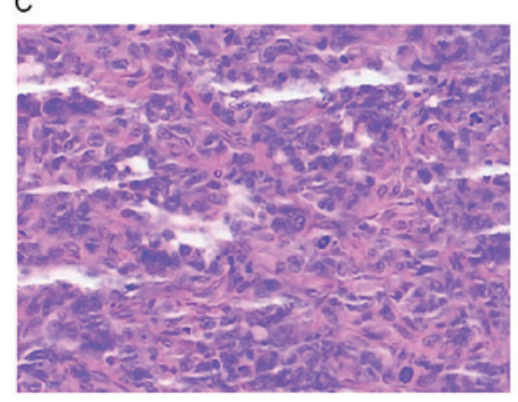

B

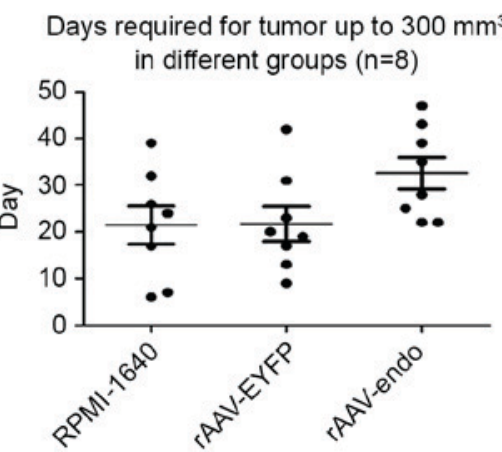

D

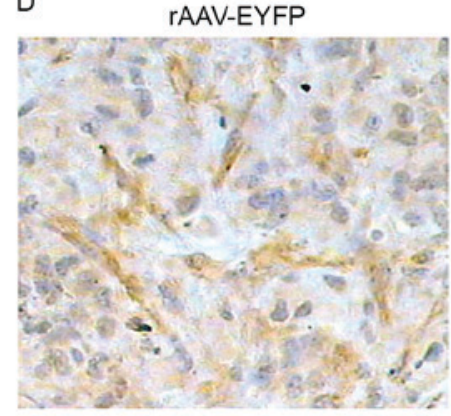

rAAV-endostatin

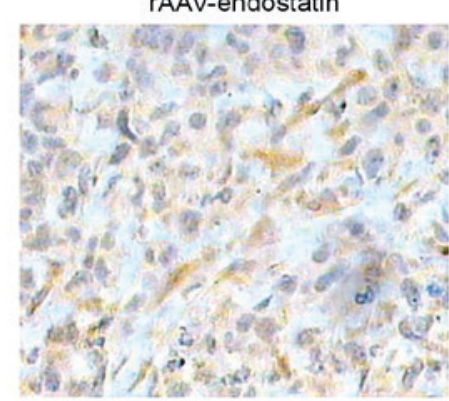

Figure 4. Therapeutic effect of rAAV-endostatin in RCC tumor. (A) The therapeutic model. (B) A total of 10 days following OR-SC-2 inoculation, intratumoral rAAV-endostatin $\left(1.0 \times 10^{11} \mathrm{v} . \mathrm{p}\right)$ or control virus injections into the established tumor were performed. The xenograft tumors with rAAV-endostatin injection took longer to reach $300 \mathrm{~mm}^{3}$ compared with the control groups: rAAV-Endostatin group, 32.63 \pm 9.75 days; rAAV-EYFP group, $21.75 \pm 10.48$ days; RPMI-1640 group, $21.50 \pm 11.42$ days. (C) Hematoxylin and eosin staining of the OS-RC-2 kidney tumor at magnification, x200. Representative images from three independent experiments were presented. (D) Subsequent to harvesting the xenograft (kidney) tumors, specimens were paraffin-embedded and cut into $5-\mu \mathrm{m}$ thick sections and processed for immunohistochemical staining of factor VIII-associated antigen. The brown areas indicate microvascular structures (magnification, $\mathrm{x} 200$ ). Representative images from three independent experiments were shown. rAAV, recombinant adenovirus-associated vector; EYFP, enhanced yellow florescent protein.

Tumor cell proliferation and apoptosis is not affected in rAAV-Endostatin-infected RCC cells. In order to investigate whether rAAV-Endostatin infection directly inhibited RCC cell growth and induce tumor cell death, RCC cells proliferation and apoptosis was analyzed. OS-RC-2 tumor cells infected with rAAV-Endostatin or control rAAV did not exhibit significant morphological changes (Fig. 2A). Tumor cell growth indicated that $\mathrm{rAAV}$-Endostatin infection did not promote or inhibit OS-RC-2 cell proliferation (Fig. 2B), and analysis of cell death following rAAV-Endostatin infection indicated that there was no significant difference in proportions of cell death compared with the control rAAV-infected cells (Fig. 2C).

Prophylactic effect of rAAV-Endostatin in RCC tumor development. In order to investigate whether vaccination of rAAV-Endostatin adenovirus inhibited RCC tumor growth, BALB/c nude mice were vaccinated with 3 doses of rAAV-Endostatin intramuscularly; $1 \times 10^{6} \mathrm{OS}-\mathrm{RC}-2$ tumor cells were subcutaneously inoculated into the BALB/c nude mice 7 days following the third immunization (Fig. 3A). The size of tumors derived from OS-RC-2 cells infected with rAAV-endostatin were smaller compared with those in the controls. rAAV-Endostatin immunization led to $50 \%$ xenograft tumors (4/8 mice) grown in nude mice whereas the control rAAV-EYFP immunization induced $100 \%$ xenograft tumor (8/8 mice) development. Monitoring of tumor growth demonstrated that vaccination of rAAV-Endostatin adenovirus significantly suppressed tumor growth comparing with control adenovirus vaccination (Fig. 3B-D).

Therapeutic effect of $r A A V$-Endostatin in RCC tumors. To additionally investigate whether rAAV-Endostatin had a therapeutic effect on the established RCC tumors, BALB/c nude mice were inoculated with $1 \times 10^{6}$ OS-RC-2 cells. A total of 10 days subsequent to tumor growth reaching $\sim 5 \mathrm{~mm}$, 3 doses of rAAV-Endostatin or control rAAV were injected into the RCC tumor (Fig. 4A). The tumor growth was monitored, and the results demonstrated that the intratumoral rAAV-Endostatin injection significantly delayed RCC tumor development. The xenograft tumors derived from RCC cells treated with intratumoral $\mathrm{rAAV}$-Endostatin injection took longer time to reach $300 \mathrm{~mm}^{3}$ compared with control cells (32.63 \pm 9.75 vs. $21.50 \pm 11.42$ and $21.75 \pm 10.48$ days, respectively; Fig. 4B). At day 60, all mice were sacrificed. The average weight of xenograft tumors with $\mathrm{rAAV}$-Endostatin injection was $0.60 \pm 0.21 \mathrm{~g}$, whereas that of xenograft tumors with the control rAAV-EYFP injection was $0.76 \pm 0.35 \mathrm{~g}$. H\&E and immunochemistry staining indicated that there was decreased angiogenesis formation in the samples that had undergone rAAV-Endostatin adenovirus immunization compared with the control rAAV-EYFP injection (Fig. 4C and D).

rAAV-Endostatin suppresses RCC tumor growth through inhibition of angiogenesis. To identify the mechanism of rAAV-Endostatin inhibition of tumor development, the 
A

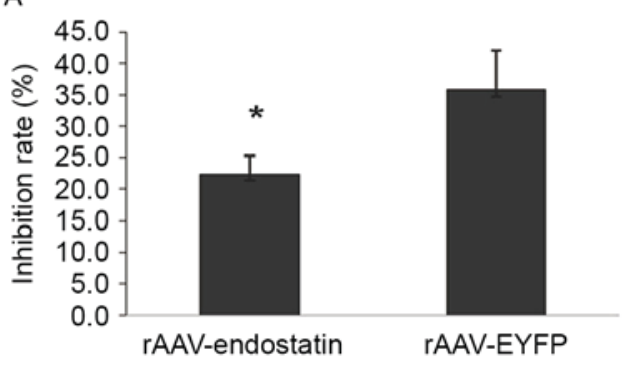

C

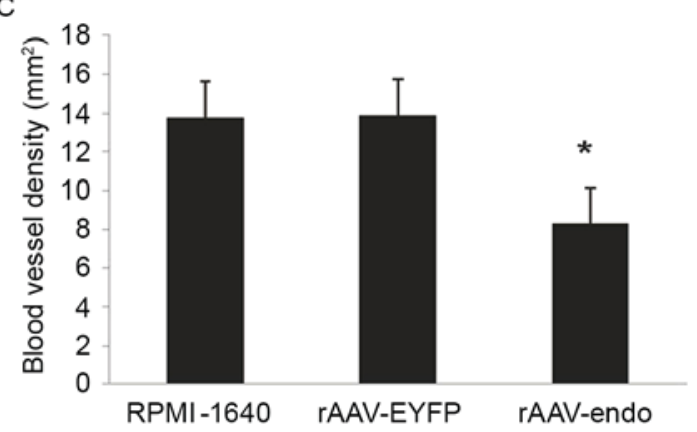

B

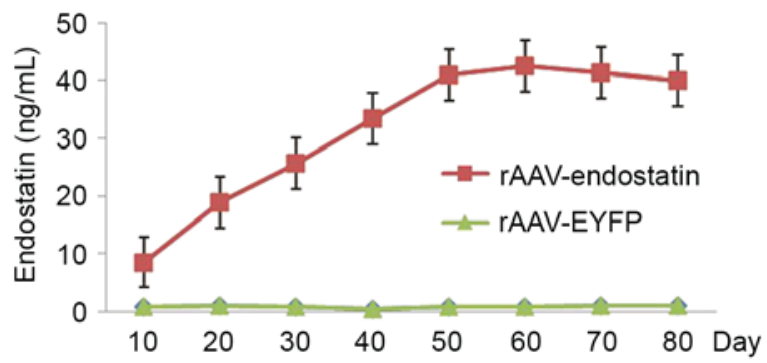

Figure 5. rAAV-endostatin suppresses RCC tumor growth through the inhibition of angiogenesis. (A) Inhibition of chemotactic activity of human umbilical vein endothelial cells treated with OS-RC-2-endostatin culture supernatant in vitro; ${ }^{*} \mathrm{P}<0.05$ vs. rAVV-EYFP. (B) Following injection of rAAV-endostatin $\left(1 \times 10^{11} \mathrm{v} . \mathrm{p}\right)$, the serum concentration of endostatin rose continuously, peaked at day 60 , and was maintained at a sustained level of $30-40 \mathrm{ng} / \mathrm{ml}$ thereafter, indicating that single use of rAAV-endostatin may lead to durative endostatin expression and secretion, and this concentration of endostatin may inhibit tumor angiogenesis effectively. (C) Following immunohistochemistry staining, the microvessel density of xenograft tumors of the group injected with rAAV-Endostatin was $8.30 \pm 3.14 / 0.739 \mathrm{~mm}^{2}$ whereas those of the control groups were $13.87 \pm 4.09 / 0.739 \mathrm{~mm}^{2}(\mathrm{rAVV}-\mathrm{EYFP})$ and $13.76 \pm 3.50 / 0.739 \mathrm{~mm}{ }^{2}$ $\left(\right.$ RPMI-1640) $(\mathrm{n}=8)$. The result suggests that single injection of rAAV-endostatin may inhibit tumor angiogenesis. ${ }^{*} \mathrm{P}<0.05$ vs. rAVV-EYFP or RPMI1640. rAAV, recombinant adenovirus-associated vector; EYFP, enhanced yellow florescent protein.

A

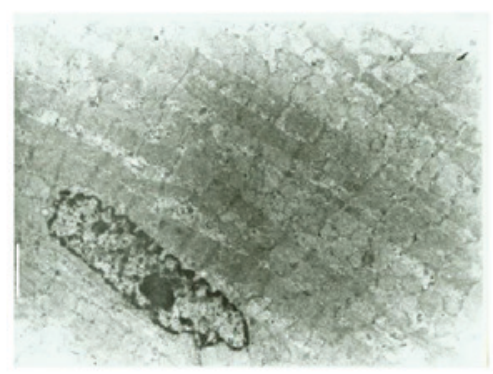

C

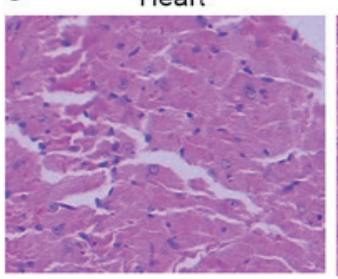

Brain

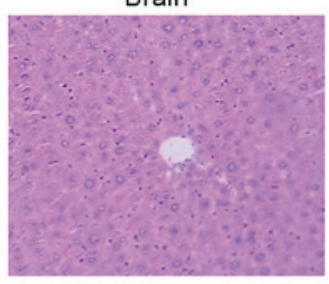

B
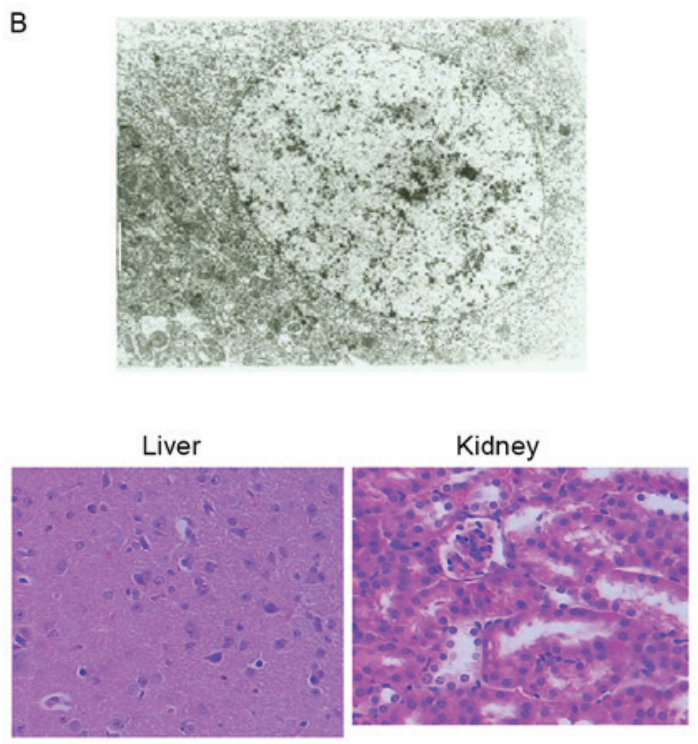

Figure 6. Transmission electron microscopy and H\&E assay of mouse vital organ following rAAV-endostatin inoculation. A total of 8 weeks following injection of 1.0x $10^{11}$ v.p rAAV-endostatin, the heart and encephalon tissues were collected for safety examination (to ensure there were no off-target effects of the virus infection on other tissues), and no adverse effect was identified in either (A) heart tissue (magnification, $\mathrm{x} 4,000$; white scale bar=2 $\mu \mathrm{m}$ ) or (B) encephalon tissue (magnification, $\mathrm{x} 4,000$; white scale bar=2 $\mu \mathrm{m}$ ) under electronic microscope. (C) H\&E staining (magnification, x200) of heart, brain, liver and kidney tissues did not exhibit significant changes in morphology. H\&E, hematoxylin and eosin.

chemotactic activity of HUVEC cells exposed to the supernatant of rAAV-Endostatin-infected OS-RC-2 cells was investigated in vitro. HUVEC chemotactic movement was inhibited by OS-RC-2-rAAV-Endostatin cell supernatant in Transwell assay $(22.40 \pm 2.97)$ compared with the control
rAAV-EYFP injection group (35.81 \pm 6.22 cells), and the inhibitory rate was $37.45 \%$ (Fig. 5A). The results indicated that endostatin was sufficient to suppress angiogenesis. Subsequent to injection of rAAV-Endostatin ( $\left.1 \times 10^{11} \mathrm{v} . \mathrm{p}\right)$, the serum concentration of endostatin rose continuously, peaked at day 60 and 
was maintained at a sustained level of $30-40 \mathrm{ng} / \mathrm{ml}$ thereafter (Fig. 5B).

Furthermore, the MVD of xenograft tumors from the rAAV-Endostatin immunization group was $8.30 \pm 3.14 / 0.739 \mathrm{~mm}^{2}$, whereas those of the control groups were $13.87 \pm 4.09 / 0.739$ (control rAAV-EYFP) and $13.76 \pm 3.50 / 0.739 \mathrm{~mm}^{2}$ (RPMI-1640), respectively (Fig. 5C). This observation suggested that the growth inhibition of xenograft tumors by rAAV-Endostatin may be through the inhibition of angiogenesis. Heart and encephalon tissues and other vital organs (brain, liver and kidney) did not exhibit any pathological changes under microscopy or transmission electron microscopy following rAAV-Endostatin injection (Fig. 6).

\section{Discussion}

Endostatin has been studied for a number of years, due to its potent anti-angiogenesis effect (7). Although the majority of therapeutic investigations have managed to utilize the purified endostatin protein, there are several limitations associated with its use: Previously, recombinant human-endostatin for clinical use was produced primarily from Escherichia coli or yeast, and therefore incapable of expressing its complete activity as in body; also, the protein purification process may denature endostatin and the purification yield is often low (23). In addition, the requirement to deliver angiogenesis inhibitors including endostatin for an extended period of time may lead to practical difficulties in clinical settings. As a protein drug, endostatin has a short half-life time in vivo and therefore requires repeated application to maintain the required therapeutic serum levels (23). As viral-based delivery systems have advantages for the durable expression of a transgene following a single dose (24), viral delivery of endostatin may be a promising strategy for tumor treatment.

The optimal vector for gene therapy would deliver therapeutic amount of transgene for a long time. In addition, the vector should be safe and poorly immunogenic, to allow multiple applications as required. Furthermore, the vector should be appropriate for the effective delivery of a transgene in a defined type of tissues. For these reasons, an adeno-associated viral vector was applied in the present study. As renal cancer growth is angiogenesis-dependent, and may also secret basic fibroblast growth factor that facilitates rAAV infection $(25,26)$, renal cancer serves as a suitable model for antiangiogenic gene therapy.

As endostatin inhibits tumor-induced vascularization only in the extracellular matrix (27), the present study adopted the IgG signal peptide to mediate endostatin secretion. For viral packaging, a co-transfection method with pAAV-Endo, pRC and pHelper vectors was applied. By using a pHelper vector that contained adeno VA, E4 and E2A regions, adenoviral contamination was avoided. Subsequent to infection of the RCC cells with rAAV-Endostatin, the IgG-Endostatin sequence was inserted into the cell genome. Endostatin was then expressed and secreted. According to the results, endothelial cell chemotactic movement was inhibited effectively, suggesting that the endostatin concentration was sufficient to suppress angiogenesis. In fact, a previous study demonstrated that even a low concentration of endostatin $(0.1 \mu \mathrm{g} / \mathrm{ml})$ was able to achieve an inhibitory effect (28). In the prophylactic and therapeutic RCC models, inoculation of rAAV-Endostatin inhibited xenograft tumor formation. These observations suggested that increased endostatin secretion in tumor environment may suppress angiogenesis, leading to the inhibition of tumor growth. It was also observed that rAAV-Endostatin injection led to a high serum level of endostatin. The MVD assay of the xenografts verified that the level of endostatin was able to inhibit tumor angiogenesis. Therefore, inoculation of rAAV-Endostatin may inhibit tumor neoangiogenesis and subsequent tumor growth. No adverse effects in heart and encephalon tissues following rAAV-Endostatin use were observed, suggesting that rAAV-Endostatin is safe and may be considered for use in a clinical setting. In conclusion, the biologically-active rAAV-Endostatin has been developed and demonstrated to be effective for the inhibition of renal tumor angiogenesis and growth. The rAAV-Endostatin appears to be safe for general use as a gene therapy agent against renal cancer.

\section{Acknowledgements}

Not applicable.

\section{Funding}

No funding was received.

\section{Availability of data and materials}

The datasets generated and analyzed in the present study are included in this published article.

\section{Authors' contributions}

SE and HR performed the experiments and analyzed the data. SE and LB designed the study and co-wrote the manuscript.

\section{Ethics and consent to participate}

This study has been approved by the ethnics committee of Tianjin Medical Hospital and written informed consent was obtained from all participants.

\section{Patient consent for publication}

The study participants provided consent for the data and any associated images to be published.

\section{Competing interests}

The authors declare that they have no competing interests.

\section{References}

1. Folkman J: Seminars in medicine of the Beth Israel Hospital, Boston. Clinical applications of research on angiogenesis. New Engl J Med 333: 1757-1763, 1995.

2. Kerbel R and Folkman J: Clinical translation of angiogenesis inhibitors. Nat Rev Cancer 2: 727-739, 2002. 
3. Fidler IJ and Ellis LM: The implications of angiogenesis for the biology and therapy of cancer metastasis. Cell 79: 185-188, 1994.

4. El-Kenawi AE and El-Remessy AB: Angiogenesis inhibitors in cancer therapy: Mechanistic perspective on classification and treatment rationales. Brit J Pharmacol 170: 712-729, 2013.

5. Burke PA and DeNardo SJ: Antiangiogenic agents and their promising potential in combined therapy. Crit Rev Oncol Hemat 39: 155-171, 2001.

6. Liekens S, De Clercq E and Neyts J: Angiogenesis: Regulators and clinical applications. Biochem Pharmacol 61: 253-270, 2001.

7. OReilly MS, Boehm T, Shing Y, Fukai N, Vasios G, Lane WS, Flynn E, Birkhead JR, Olsen BR and Folkman J: Endostatin: An endogenous inhibitor of angiogenesis and tumor growth. Cell 88 : 277-285, 1997.

8. Lim J, Duong T, Lee G, Seong BL, El-Rifai W, Ruley HE and Jo D: The effect of intracellular protein delivery on the anti-tumor activity of recombinant human endostatin. Biomaterials 34 : 6261-6271, 2013

9. Blezinger P, Wang JJ, Gondo M, Quezada A, Mehrens D, French M, Singhal A, Sullivan S, Rolland A, Ralston R and Min W: Systemic inhibition of tumor growth and tumor metastases by intramuscular administration of the endostatin gene. Nat Biotechnol 17: 343-348, 1999.

10. Ma HI, Lin SZ, Chiang YH, Li J, Chen SL, Tsao YP and $\mathrm{Xiao} \mathrm{X}$ : Intratumoral gene therapy of malignant brain tumor in a rat model with angiostatin delivered by adeno-associated viral (AAV) vector. Gene Ther 9: 2-11, 2002.

11. Ma HI, Guo P, Li J, Lin SZ, Chiang YH, Xiao X and Cheng SY: Suppression of intracranial human glioma growth after intramuscular administration of an adeno-associated viral vector expressing angiostatin. Cancer Res 62: 756-763, 2002.

12. Daya $\mathrm{S}$ and Berns KI: Gene therapy using adeno-associated virus vectors. Clin Microbiol Rev 21: 583-593, 2008.

13. Ponnazhagan S, Curiel DT, Shaw DR, Alvarez RD and Siegal GP: Adeno-associated virus for cancer gene therapy. Cancer Res 61: 6313-6321, 2001

14. Davidoff AM, Nathwani AC, Spurbeck WW, Ng CY, Zhou J and Vanin EF: rAAV-mediated long-term liver-generated expression of an angiogenesis inhibitor can restrict renal tumor growth in mice. Cancer Res 62: 3077-3083, 2002.

15. Shi WY, Teschendorf C, Muzyczka N and Siemann DW Adeno-associated virus-mediated gene transfer of endostatin inhibits angiogenesis and tumor growth in vivo. Cancer Gene Ther 9: 513-521, 2002.

16. Shi W, Teschendorf C, Muzyczka N and Siemann DW: Gene therapy delivery of endostatin enhances the treatment efficacy of radiation. Radiother Oncol 66: 1-9, 2003.

17. Ponnazhagan S, Mahendra G, Kumar S, Shaw DR, Stockard CR, Grizzle WE and Meleth S: Adeno-associated virus 2-mediated antiangiogenic cancer gene therapy: Long-term efficacy of a vector encoding angiostatin and endostatin over vectors encoding a single factor. Cancer Res 64: 1781-1787, 2004.
18. Noro T, Miyake K, Suzuki-Miyake N, Igarashi T, Uchida E, Misawa T, Yamazaki Y and Shimada T: Adeno-associated viral vector-mediated expression of endostatin inhibits tumor growth and metastasis in an orthotropic pancreatic cancer model in hamsters. Cancer Res 64: 7486-7490, 2004.

19. Subramanian IV, Ghebre R and Ramakrishnan S: Adenoassociated virus-mediated delivery of a mutant endostatin suppresses ovarian carcinoma growth in mice. Gene Ther 12: 30-38, 2005.

20. Chen H: Comparative observation of the recombinant adeno-associated virus 2 using transmission electron microscopy and atomic force microscopy. Microsc Microanal 13: 384-389, 2007.

21. Justus CR, Leffler N, Ruiz-Echevarria M and Yang LV: In vitro cell migration and invasion assays. J Vis Exp, Jun 1, 2014. doi: $10.3791 / 51046$

22. Puri TS, Shakaib MI, Chang A, Mathew L, Olayinka O, Minto AW, Sarav M, Hack BK and Quigg RJ: Chronic kidney disease induced in mice by reversible unilateral ureteral obstruction is dependent on genetic background. Am J Physiol Renal Physiol 298: F1024-F1032, 2010.

23. Mohajeri A, Sanaei S, Kiafar F, Fattahi A, Khalili M and Zarghami N: The challenges of recombinant endostatin in clinical application: Focus on the different expression systems and molecular bioengineering. Adv Pharm Bull 7: 21-34, 2017.

24. Katz MG, Fargnoli AS, Williams RD and Bridges CR: Gene therapy delivery systems for enhancing viral and nonviral vectors for cardiac diseases: Current concepts and future applications. Hum Gene Ther 24: 914-927, 2013.

25. OBrien T, Cranston D, Fuggle S, Bicknell R and Harris AL: Two mechanisms of basic fibroblast growth factor-induced angiogenesis in bladder cancer. Cancer Res 57: 136-140, 1997.

26. Nguyen JT, Wu R, Clouse ME, Hlatky L and Terwilliger EF: Adeno-associated virus-mediated delivery of anti-angiogenic factors as an antitumor strategy. Hum Gene Ther 10: 839-839, 1999.

27. Kim YM, Jang JW, Lee OH, Yeon J, Choi EY, Kim KW, Lee ST and Kwon YG: Endostatin inhibits endothelial and tumor cellular invasion by blocking the activation and catalytic activity of matrix metalloproteinase. Cancer Res 60: 5410-5413, 2000.

28. Dhanabal M, Ramchandran R, Volk R, Stillman IE, Lombardo M, Iruela-Arispe ML, Simons M and Sukhatme VP: Endostatin: Yeast production, mutants, and antitumor effect in renal cell carcinoma. Cancer Res 59: 189-197, 1999.

This work is licensed under a Creative Commons

Attribution-NonCommercial-NoDerivatives 4.0 International (CC BY-NC-ND 4.0) License. 